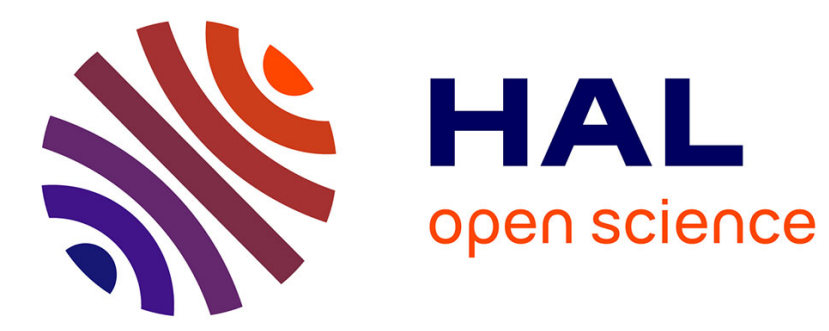

\title{
Frequency-amplitude behavior in the incipient movement of grains under vibration
}

\author{
K.A. Valenzuela Aracena, J.G. Benito, Luc Oger, I. Ippolito, R.O. Uñac, A.M. \\ Vidales
}

\section{- To cite this version:}

K.A. Valenzuela Aracena, J.G. Benito, Luc Oger, I. Ippolito, R.O. Uñac, et al.. Frequency-amplitude behavior in the incipient movement of grains under vibration. Particuology , 2018, 40, pp.1-9. 10.1016/j.partic.2017.11.009 . hal-01874675

\section{HAL Id: hal-01874675}

\section{https://hal-univ-rennes1.archives-ouvertes.fr/hal-01874675}

Submitted on 18 Sep 2018

HAL is a multi-disciplinary open access archive for the deposit and dissemination of scientific research documents, whether they are published or not. The documents may come from teaching and research institutions in France or abroad, or from public or private research centers.
L'archive ouverte pluridisciplinaire HAL, est destinée au dépôt et à la diffusion de documents scientifiques de niveau recherche, publiés ou non, émanant des établissements d'enseignement et de recherche français ou étrangers, des laboratoires publics ou privés. 


\title{
Frequency-amplitude behavior for incipient movement of grains under vibration
}

Karina A. Valenzuela Aracena ${ }^{\mathrm{a}}$, Jesica G. Benito ${ }^{\mathrm{a}}$, Luc Oger ${ }^{\mathrm{b}}$, Irene Ippolito ${ }^{\mathrm{c}}$, Rodolfo O. Uñac $^{\mathrm{a}}$, Ana M. Vidales ${ }^{\mathrm{a}^{*}}$

${ }^{a}$ INFAP, CONICET, Departamento de Física, Facultad de Ciencias Físico Matemáticas y Naturales, Universidad Nacional de San Luis, Ejército de los Andes 950, D5700HHW, San Luis, Argentina

${ }^{b}$ Institut de Physique de Rennes, UMR UR1-CNRS 6251, Université de Rennes 1, 263,Avenue du Général Leclerc, Campus de Beaulieu, CS 74205, 35042 Rennes Cedex, France

${ }^{c}$ Universidad de Buenos Aires, Facultad de Ingeniería, Grupo de Medios Porosos, Paseo Colón 850, 1063, Buenos Aires, Argentina

* corresponding author, avidales@unsl.edu.ar

\begin{abstract}
This paper focus on the onset of the movement of particles placed on a horizontal rough surface subjected to a vertical sinusoidal vibration. We track the problem through experiments, theory and numerical simulations. The frequencies needed to put particles in movement decays exponentially with the input amplitude of the oscillations. This behavior is explained through a simple approach where a forced damped harmonic oscillator is used with a spring constant representing all the interactions between the particle and the surface. The numerical results compare well with the experimental ones, proving that the forces included in the numerical calculations are suitable to account for the main aspects of the problem, even though that the complexity of the surface is not fully accounted for. Describing the way in which the frequency varies with amplitude could be relevant when technological applications like, for instance, surface cleaning take place.
\end{abstract}

Keywords: vibration, incipient movement, forced oscillator, 3D DEM simulation 


\section{Introduction}

The displacement of grains in industrial processes frequently implies external energy supply through agitation in conveyor belts, truck transport, drilling operations, among many others. As a result, the manipulation of granular matter always carries secondary effects, desired or not. Segregation, crushing, jamming, flow fluctuations, particle resuspension and saltation are just some examples. Much scientific attention is dedicated to the solution of the problems related to granular materials behavior, but many questions, especially those related to the microscopic interaction between grains and surfaces, are not yet solved.

It has been proven that the initiation of movement of particles deposited on a surface is crucial to predict their possible further resuspension by air flow turbulence (Henry \& Minier (2014); Reeks \& Hall (2001); Valenzuela Aracena et al. (2017)). The concept of “fluid threshold” (or the "aerodynamic threshold”) is already introduced by Bagnold many years ago (Bagnold, R.A. (1941); Chepil (1945); Zingg (1953)). On the other hand, initial destabilization of sand grains by the wind is important to answer if transport of sandy matter in desert geography can be expected (Oger \& Valance (2017)).

In the same way, vibration, as a perturbation of particle’s equilibrium, is relevant in many theoretical and applied problems, like, for instance, the removal of small particles from surfaces in engineering applications (Ziskind, Fichman \& Gutfinger (2000)). Likewise, in granular matter manipulation stages, the mechanical vibration of grains, either horizontal or vertical, is also an important external perturbation.

Naturally, if the vertical vibration can compensate the gravitational acceleration, jumps are observed as typical movements for the grains. Studies of the fluidization of granular beds subjected to vibrations are commonly found in the literature (Eshuis et al. (2007), Renard et 
al. (2001), Mawatari et al. (2002)), where the critical intensity of the external excitations to fluidize the grains is investigated. Moreover, even jamming of a drained granular medium can be observed depending on the time needed for rearrangements of the grains ( $\mathrm{D}^{\prime}$ Anna \& Gremaud (2001)). In recent years, the formation of patterns on the surface of vibrated layers of grains has been extensively studied. Depending on the whole set of parameters (amplitude and frequency of the excitation, shape and size of grains and container, and so on) different kinds of standing waves can be observed, with varied textures (Melo, Umbanhowar \& Swinney (1994); Melo, Umbanhowar \& Swinney (1995); Metcalf, Knight \& Jaeger (1997)). On the other hand, basic studies concerning the excitation of small spheres deposited on a horizontal platform has been conducted in the past (Ripperger \& Hein (2005); Tippayawong \& Preechawuttipong (2011)).

The first step to describe a system consisting of a particle sitting on a vibrating surface can be performed considering a mechanical approach as the one presented in Dybwad (1985). In that work, the author studies the problem of a particle of mass $m$ deposited on a vibrated surface (quartz resonator) as two coupled springs. The particle is linked to the surface through an elastic force with constant $k$. The quartz resonator has a mass $M$ and an elastic constant $K$, and its resonance frequency only depends on its elastic properties. The frequency of the coupled system (particle - quartz resonator) results to depend on the elastic force constant, $k$, of the particle. The behavior of that frequency is found to contradict the mass loading expectation, i.e., that the frequency of an oscillating system will decreases when its mass increases. An experimental estimation of the value of the elastic constant shows that the actual area of contact of the two touching solids (particle and surface) is very much less than their geometrical area, i.e., than the area of contact that could be determined 
through a direct calculation, given that it may be lowered by the presence of roughness on the surfaces (Dybwad (1985)).

As a second step in the description of the perturbation of a particle deposited on a surface, several linear and nonlinear oscillation models are introduced and analyzed in Ziskind, Fichman \& Gutfinger (2000) to demonstrate whether particle removal is possible for soft and hard particles on smooth and rough surfaces. One of the conclusions in that paper is that the natural frequency may be reduced by up to two orders of magnitude in case that the distance between two contacts at which the particle touches the surface is much smaller than the particle radius. Besides, they emphasize the idea that behavior of particles is like if they were linked to the surface by springs (Dabros, Warszynsky\& van de Ven (1994); Ziskind, Fichman \& Gutfinger (2000)).

Experiments using aerosol particles deposited on a vibrating glass surface demonstrate that the wall vibration plays two opposite roles, i.e., to increase the separation force between particles and the wall, and to increase the particle deposition rate due to active capture of aerosol particles (Theerachaisupakij et al. (2002)). In the same way, analytical models show that frequency of particle-surface interaction is found to significantly influence the removal rate of micro-particles from a vibrating surface (Tippayawong \& Preechawuttipong (2011)). Besides, the description of the re-entrainment process implies the analysis of adhesion and capillary forces between the particle and the surface, the inertia of the particle when accelerated and its contact interaction with the particles belonging to the surface, especially in cases where roughness is of the order of the size of the beads deposited on it. For that reason, the vibration of a surface as an indirect method for the measurement of adhesion forces between a particle and a wall is used in Hein et al. (2002), by correlating particle re-entrainment events with the acting acceleration. 
To conclude, we may say that the dynamic involved in the initiation of a particle movement on a surface subjected to an external perturbation is not simple, and the inclusion of all the parameters playing a role is not straightforward (Mullins et al. (1992)), even when only a threshold velocity for movement initiation is looked for (Soepyan et al. (2016)). As a result, the conditions under which the incipient movement of grains subjected to vibration is expected are still an open-question topic, even for particles of the millimeter sizes.

The aim of the present study is to predict the critical conditions for a particle to move when it rests on a vibrating surface, and to evaluate the physical parameters involved in this process. To pursue this objective we perform series of experiments over rough surfaces (built with micrometer glass beads) where different particle sizes (within the millimeter range) are deposited for vibration. We also develop a theoretical approach that describes the main aspects of the experimental behavior found here. The idea behind is to find the critical frequency-amplitude pairs needed to initiate the movement of the deposited grains for different bead sizes and materials and to prove that the system behaves like a forced damped oscillator with a weak elastic constant (compared to that of the particle's material) given by the force interaction between particle and surface. In this sense we follow the former ideas developed in the works by Dybwad (1985) and Ziskind, Fichman \& Gutfinger (2000).

To reinforce our goal, we also perform some numerical tests using a Discrete Element Method with the appropriate interactions between the grains and the surface, to model the experimental conditions (Herrmann \& Luding (1998); Luding (1998)). Indeed, we simulate a set of experimental results where a good agreement is found when we include the presence of elastic forces due to the bead material and also those due to adhesion and capillary effects. 


\section{Experiments}

\subsection{Experimental setup and materials}

The device for the experiments consists of the sinusoidal vertical vibration of a horizontal rough surface on which beads are placed in order to study the onset of their movement. The rough surface is built by gluing glass beads on a flat circular plate with a diameter of $6 \mathrm{~cm}$. We work with two different rough surfaces: one, made with $250 \mu \mathrm{m}$ glass beads and the other one of $500 \mu \mathrm{m}$ glass beads, with respective size dispersions of $44 \mu \mathrm{m}$ and $85 \mu \mathrm{m}$. A Scanning Electron Microscope (SEM) image is shown in Figure 1 to illustrate the surface topography created by the glued beads for the case of $250 \mu \mathrm{m}$. It is easy to see in some regions, 2 or 3 layers of beads due to the manufacturing method of the surface. This is also observed in the $500 \mu \mathrm{m}$ surface.

Concerning the free particles deposited on the surface, we use glass beads whose mean diameters are: $(0.93 \pm 0.02) \mathrm{mm},(1.98 \pm 0.04) \mathrm{mm},(3.26 \pm 0.09) \mathrm{mm}$ and $(3.98 \pm 0.03)$ $\mathrm{mm}$; and stainless steel ball bearings of 2, 3 and $4 \mathrm{~mm}$.

The plate is fixed to the drive arm of a mechanical oscillator and can vibrate with a selected frequency given by a wave generator connected to it. The mechanical oscillator has a drive arm that allows fixing on it the plate with the rough surface to be vibrated. The amplitude of oscillation of this arm is proportional to the signal (measured in volts) of the wave generator connected to the mechanical oscillator. The linear correspondence between voltage and amplitude is verified and calibrated carefully. To this end we make use of two different techniques. The first one consists in the tracing, with the help of a fine fiber pen, of the trajectory of the oscillating arm-plate system. The double check is achieved by 
recording the movement of the arm-plate system with a video camera Casio EXILIM EXZR400.

We also verify the dependence of the amplitude given by the generator on the different frequency ranges and that the sinusoidal shape of the signal does not change with time. For the range involved in the present experiments, constant amplitude can be provided, independently of the working frequency.

Another important point is to know if the critical frequency depends on the rate of increment during the experimental run. Indeed, we detect a dependence on the frequency rate, so a controlled ramp is provided with a fixed rate equal to $0.63 \mathrm{rad} / \mathrm{s}^{2}$ in all the experiments.

In the present experiments we use sinusoidal waves with a given well controlled amplitude, $A$, and frequency, $\omega$. Figure 2 gives a sketch of the experimental set up. The values selected and measured for the amplitudes used in the experiments are: $0.73,1.28,1.91,2.28,2.58$, 2.87, 3.12, 3.47, 3.88 and $4.03 \mathrm{~mm}$, with an error of $\pm 0.06 \mathrm{~mm}$. The error in the different frequencies provided by the generator is of the order of $1 \mathrm{rad} / \mathrm{s}$.

Initially, ten spheres with a given diameter are deposited randomly over the surface. They are carefully separated to prevent possible contacts or hits among them or with the lateral edges which are transparent thin walls stuck around the disk to avoid the flow out of the free particles.

The desired amplitude is selected and fixed in the wave generator (see (1) in Figure 2) and the platform is subjected to an oscillation starting from a frequency of $0.10 \mathrm{~Hz}$ (approximately $0.63 \mathrm{rad} / \mathrm{s}$ ). Next, the frequency is increased in fixed steps at a rate of 0.10 Hz/s. 
With the help of a magnifying glass, we check for the incipient movement of particles and record the critical frequency, $\omega_{c}$, at which at least half of the deposited spheres starts to move from their original positions. Movement is considered when a particle has a displacement of the order of its radius. This procedure is also recorded by the digital video camera referred above, at $140 \mathrm{fps}$, to complete the determination of $\omega_{\mathrm{c}}$ (see (5) and (6) in Figure 2). Once the critical frequency is reached, the experimental run ends, and the oscillator is turned off.

A new value for the amplitude of oscillation is chosen and the spheres are redistributed over the surface. The frequency is set to $0.10 \mathrm{~Hz}$ and the excitation is started again with the same increasing rate. The critical frequency for the new amplitude value is registered in the same way as explained before.

To avoid important humidity effects, experiments are always performed with a relative ambient humidity between 35\% and 55\%. This allows having the same capillary interaction between the spheres and the surface (Kohonena (2004); Rabinovich et al. (2002)).

Finally, for a given surface and a given deposited sphere size and material, a set of 200 different positions on the surface are inspected in order to average the results shown in the next section.

\subsection{Results and discussion}

In our experiments, we observe that positions of the beads which initiate their motion are randomly distributed on the surface. In Figure 3 we show the results obtained for the critical frequencies, $\omega_{\mathrm{c}}$, needed to put into incipient movement at least $50 \%$ of the glass 
particles deposited on the vibrating surface as the amplitude of the excitation is increased. The error bars for the amplitude are indicated and those for the statistical error in the determination of the critical frequency are of the order of the symbol size. Part (a) of the figure corresponds to the $250 \mu \mathrm{m}$ surface and part (b) to $500 \mu \mathrm{m}$. The different ratios between the size of the free spheres and the size of the beads belonging to the surface are indicated in each case.

On the other hand, our results demonstrate to be practically independent of the size of the beads used to build the surface. This implies that the roughness of the surface created by the glued spheres is such that the characteristic length of it is not dictated by the size of the constituting beads, but rather by the geometrical arrangement of them with the glue. In other words, the topography of the surface created by the glued grains masks the influence of their own sizes.

According to the definition of $\omega_{\mathrm{c}}$, the set of points divides the upper region corresponding to the existence of movement of at least $50 \%$ of thespheres from the lower part with absence of movement or with incipient movement of less than $50 \%$ of the particles.

The results for both surfaces and all ratios are quite similar. They can be described by an exponential decay in all cases, independently of the size ratio. So, as the amplitude of the oscillation increases, the critical frequency needed to initiate the particle's movement decreases.

In order to know whether the behavior is still the same for a different material, we perform a series of experiments with stainless steel spheres deposited on the same two rough surfaces. By following the same procedure explained earlier, we obtain the results shown in Figure 4. Comparing the two parts of the figure, it is clear that the behavior for both 
surfaces is practically the same, with a slight displacement of the data to higher frequencies for the smaller spheres. As indicated by the curves, the behavior can also be fitted by an exponential decay. It is necessary to note that experiments with the smaller $1 \mathrm{~mm}$ stainless steel beads are not performed to avoid static charge effects.

According to the results shown so far, a clear dependence on the excitation amplitude is experimented by the critical frequency needed for a given particle to start its motion. In the scheme that the system could behave like a lightly damped forced oscillator, it is expected that one natural resonance frequency will be present. Nevertheless, the exponential decay of the critical frequency as the vibration amplitude increases is very solid and, as we will see in the next section, the simple scheme of a forced oscillator has to be rethought to explain our present results (Ziskind, Fichman \& Gutfinger (2000)).

\section{Theoretical approach}

\subsection{Model of the results}

In this section, we explain, from a theoretical point of view, the possible physical basis leading to the experimental results presented above. Using a basic approach, we analyze the problem of a spherical particle on top of a vibrating bed. This system can be represented as a forced damped harmonic oscillator like the one sketched in Figure 5.

As presented in the introduction, we assume that the key point is to consider that our system behaves like a harmonically excited oscillator with a spring representing the effective interactions between particle and surface. These interactions are due to the intermolecular forces and capillary forces, which lead us to assume that their effect is like 
an elastic bond ("spring”) between the particle and the surface, with stiffness constant $k$ (Ziskind, Fichman \& Gutfinger (2000)).

In this way, the stiffness constant represents the effective bond seen by the excited particles. Here, it is important to clarify that the attractive part of the effective interaction force in our model could be attributed to the presence of van der Waals and capillary effects. Indeed, these forces are still important when compared with gravitational ones even in the case of particles with few millimeters size. Both forces increase with the radius of the particle and it has been calculated that their value are not negligible respect to the weight (Israelachvili (1991), Zhu et al. (2007)). For capillary forces, although the relative humidity values at which experiments are performed is not so high, it is enough to generate capillary bridges among the particles and the surface that have to be considered in the problem (Herminghaus (2005)). On the other hand, were these forces not present, the sole inertial force due to the external excitation would yield to a constant critical acceleration, which is not the case, as will be shown below.

Like it is clearly discussed in Ziskind et al. (2000), the oscillatory motion of a particle on a surface may be caused by the mechanical vibrations of it and nonlinear effects can be present. The fact that the oscillation of a rough surface can cause a complex vibration movement of the particle deposited on it makes it necessary to consider a nonlinear behavior. In the case of a forced oscillator with a nonlinear restitution force (Pain, H.J. (1976)) it is calculated that, depending on the sign of the nonlinear term, the effective spring constant decreases with the deformation of the spring, which is propose in our model below.

It is very difficult to measure the elastic properties of the bond between particles and surface but, our experimental results show (as plotted further below) that a non linear 
behavior is present. Given the complexity of resolving the equations of motion for such a nonlinear behavior (Wang L. (1999)), we propose an approximation by considering a simple linear harmonic damped oscillator where we introduce a correction on the dependence of the natural frequency on the oscillation amplitude (Ziskind, Fichman \& Gutfinger (2000)).

We assume an external harmonic force exerted on the particle through the spring by the vibrating bed with amplitude $A$ and frequency $\omega / 2 \pi$. Using standard oscillation analysis and with the help of the sketch in Figure 5, let figure out which is the amplitude that the particle will see when external excitation is turned on.

To follow the theoretical model proposed here, one only has to remember the main features of a forced damped harmonic oscillator. Thus, the amplitude seen by the particle can be calculated following the standard resolution as:

$$
A^{*}(\omega)=\frac{F_{0} / m}{\sqrt{\left(\omega_{0}^{2}-\omega^{2}\right)^{2}+(\gamma \omega)^{2}}}
$$

In equation (1), $\omega_{0}$ and $\gamma$ are the natural frequency of the system and the damping constant of the elastic bond between the particle and the surface, respectively. As known, $\omega_{0}$ will depend on the stiffness of the bond.

In the present case, experiments are done by varying the frequency of the external excitation, so the amplitude of the force, $F_{0}$, is not a constant but varies with $A \omega^{2}$, where $A$ is the input amplitude of the oscillator, fixed at each experimental run. Thus, as the run goes on, the amplitude seen by the sphere is: 


$$
A^{*}(\omega)=\frac{A \omega^{2}}{\sqrt{\left(\omega_{0}{ }^{2}-\omega^{2}\right)^{2}+(\gamma \omega)^{2}}}
$$

Using equation (2), the acceleration undergone by the particle can be calculated as $A^{*}(\omega) \omega^{2}$. When a different value for the amplitude $A$ is selected (a new experimental run begins by increasing the frequency) new series of $A^{*}(\omega)$ values are seen by the particle as the experiment goes on and, as a consequence, the velocity and the acceleration suffered by the particle change.

To illustrate our point we apply the theoretical model to two of the experiments performed above. We consider glass spheres of $1 \mathrm{~mm}$ deposited on the $250 \mu \mathrm{m}$ and on the $500 \mu \mathrm{m}$ glass beads surfaces. Figure 6 shows the forces undergone by the particle deposited on the oscillating surface for each value of the amplitude and calculated by using $F^{*}=$ $m A^{*}(\omega) \omega^{2}$. Note that in our present model, the curves will be the same for both types of surfaces. The mass of the sphere is $1.053 \times 10^{-6} \mathrm{~kg}$, by taking $2500 \mathrm{~kg} / \mathrm{m}^{3}$ as the glass density. For a better visualization of the values we plot the results in a semi-log scale. The meaning of the horizontal lines (very close one to the other) is explained below.The ten curves correspond to the different amplitudes of excitation used in the experiments and they are enumerated in the legend. Besides, a damping constant of $200 \mathrm{~s}^{-1}$ is considered. This value will be (as seen below) of the order of the natural frequencies entering in equation 2, thus modeling a lightly damped harmonic oscillator. In our checks, the model shows to be almost insensitive to the value of this constant starting at $100 \mathrm{~s}^{-1}$ and up to one order of magnitude. Only the range of oscillation frequencies involved in our experiments is displayed in the plots. 
Taking into account that some authors find that the stiffness of a spherical particle is not constant but increases with particle deformation (Ziskind, Fichman \& Gutfinger (2000)), it is not unreasonable to think that, in our present scenario, $k$ will depend on the amplitude of the oscillations. It is worth noting that it is very difficult to quantify the elastic properties of the bond between particle and surface. For that reason some assumptions and estimations are done for the values of $\omega_{0}$ used in the figures.

In our present case, it is expected that particle deformation will be larger as the applied acceleration increases and, thus, according to Ziskind, Fichman \& Gutfinger (2000), the stiffness $k$ will increases. Experimentally, the critical acceleration decreases as the input amplitude $A$ increases (see below). Consequently, $k$ decreases for increasing values of the input $A$. Given that the mass of the particles is a constant, a decrease in $k$ means a decrease in the natural frequency $\omega_{0}$ of the system. Taken into account that an exponential decay is evident among the critical frequency-amplitude pairs found in our experiments, we propose, as a reasonable trend, an exponential decay of the natural frequency with the amplitude $A$. This idea is also in coincidence with the expected decaying behavior of vibrating frequencies as the restoring force becomes non-linear (Kovacic (2011)).

Considering the range of values obtained for the experimental critical frequencies and their relation with the different input amplitudes, we propose that the natural frequencies $\omega_{0}$ obey the decaying behavior with $A$ displayed in Figure 7. It is worthy to say that these are the values for $\omega_{0}$ which are used to plot the curves in Figure 6.

Now, consider the force necessary to overcome the interaction forces holding a particle attached to the surface (spring breaking). The horizontal lines in Figure 6 represent this force, assuming that its value is constant for a given particle size. Although we do not know 
this precise value, we can say that its magnitude is of the order of the nano-Newtons for micrometric particles on flat surfaces (Ripperger \& Hein (2005)). Again, taking into account the range of values for $\omega_{c}$ found in our experiments, we are only able to estimate detaching forces of $267 \mathrm{nN}$ for $250 \mu \mathrm{m}$ and $259 \mathrm{nN}$ for $500 \mu \mathrm{m}$ surfaces. This rough estimation seems reasonable if one thinks that, in our present case, we deal with $1 \mathrm{~mm}$ particles on surfaces where the attaching forces are expected to decrease because of the presence of roughness. We will discuss these values in the next section below.

The successive intersections of the horizontal lines with the different curves in Figure 6 give the critical frequencies at which incipient movement of the particles is expected at a given amplitude of excitation. The values obtained at the intersections are plotted, respectively, in each part of Figure 8, along with the corresponding experimental results evaluated from the data in Figure 3(a) and (b). The agreement is quite good.

In the same way, taking the different values for $\omega_{c}$ obtained from the intersections, one can calculate the maximum critical acceleration, $a_{c}=A \omega_{c}{ }^{2}$, at which the vibrating plate is subjected when $50 \%$ of particle movement is starting as a function of the amplitude. The results are respectively presented for both surfaces in Figure 9, where the comparison with experimental data is also shown. Here again, the agreement is good and we can appreciate the above mentioned decreasing trend of $\omega_{0}$ as the amplitude increases.

\subsection{Discussion}

The theoretical approach developed here allows the plotting of the force seen by the moving beads as the external frequency increases, for different amplitudes. Given that in all the cases the moving particle is the same, the force calculus for both rough surfaces yields 
the same curves shown in Figure 6. On the other hand, the cohesion force (adhesion plus eventual capillary bridges) experimented by the particles when they try to detach from the surface (spring breaking) is different. The two values proposed (267 nN and $259 \mathrm{nN}$ ) for each case are very close. Such equal values are evidencing the similar behavior found when comparing Figures 3 (a) and (b). They are deduced by drawing horizontal lines in Figure 6 that take into account the experimental range of values within which $\omega_{c}$ varies with $A$. In other words, by taking into account the frequency range, the intersections of the horizontal line with each amplitude curve give the correct values of the experimental critical frequencies. We have to say that these force values in Figure 6 are just rough estimations obtained from the model. They represent the force needed to destabilize the particle and are inside the wide range of adhesion forces estimations found in the literature (Zhu et al. (2007), Ripperger \& Hein (2005), Jones et al. (2002)). Evidently, if one could measure those force values, the inverse way could be performed to predict the critical frequencyamplitude ranges originally measured in our experiments.

In this way, one could conclude that the interaction of the beads with both surfaces is quite similar and this is likely due to the way that the surfaces are constructed. The high degree of disorder present in the space distribution of the glued beads on the surface is more important that their size, thus, the moving particles interact in a similar way with both surfaces.

It is interesting to mention that in experiments for ideally rigid particles on a rigid plane which is vibrated sinusoidally, the so called Froude criterion is suitable to characterize critical detachment frequencies when only inertial forces are present. As expressed above, adhesion forces have to be taken into account. For that reason, the experimental data cannot 
be represented by that criterion and one would have to modify that criterion to embody both the elastic and adhesion properties of the particle-plate system (Eshuis et al. (2007), Renard et al. (2001)).

The model is able to describe quite well the experimental dependence of the critical frequencies on the input amplitude. Only the idea of a harmonic oscillator resonating at the natural frequency corresponding to the particle material is not enough, in coincidence with the ideas proposed by Dabros, Warszynsky \& van de Ven (1994) and by Ziskind, Fichman \& Gutfinger (2000). Although the exponential decrease of the critical frequency $\omega_{c}$ with the amplitude $A$ is not explicitly derived from the theoretical calculus, it can be appropriately deduced by the present model as soon as an appropriate fitting of the spring stiffness $\omega_{0}$ is implemented in the model.

Finally, the decrease of the acceleration as $A$ increases is an indication that a non-linear behavior of the natural frequency of the system could be happening. Indeed, the assumption for the dependence of $\omega_{0}$ on the amplitude, as displayed by Figure 7 , is necessary to explain, through this simplified model, the results obtained experimentally.

\section{Simulation Model}

In this Section we present the outline of our simulation model to show which are the main forces contributing to a good description of the problem and validating that the inclusion of elastic, adhesion and capillary forces are enough to explain, from a numerical approach, the experimental behavior found. 


\subsection{Numerical Setup}

We use a classical Discrete Element Method (DEM) model to represent the dynamical interaction between the moving particles and the rough surface. The present model follows the two-dimensional formulation of Savage (1993) and the three-dimensional extension used by Oger, Ippolito \& Vidales (2007). In order to make the reading clearer all the equations are regrouped in the Appendix.

The particles are modeled as spheres using a "soft-particle" approach (equations A1toA3), where each sphere can have multiple contacts that can persist for extended durations (typically during 50 time steps). Normal and tangential forces can develop at the contact between two spheres. When only compressive forces are allowed, the simulations represent dry, non-cohesive particle assemblies. The presence of tensile forces at the contacts models cohesive particles. In this case, short range interactions can occur up to the breakage of the adhesive forces or the breaking of a liquid bridge. No long range interactions are present in this model. In this way, bonds between contacting spheres can be introduced at the initial stage of the packing or during the oscillation process, thus mimicking an assembly of particles with tensile strengths.

In our model, a tensile (negative) value is possible up to a limiting tensile strength defined for the bond, after which the bond breaks. These bonds can arise, as expected, due to the presence of van der Waals and/or capillary forces (see equations A4 and A5). When no cohesive forces are present, a contact takes place only when two spheres overlap, and the normal and tangential forces increase as the centers of the particles approach each other.

The tangential force has also a critical tensile strength below which it is modeled as a viscoelastic one and, above it, the bond breaks and the tangential force follows the Mohr Coulomb law. 
In order to simulate local contact effects and non-perfect spherical particles, a rolling resistance parameter has been introduced recently (Ai et al. (2011)). This parameter represents the effects of the shape of the particle on its rolling ability and the possible resistance due to plastic deformation around the contact and/or possible viscous hysteresis. We consider this parameter in our model and its value is taken between the range 0 (no effect) to 0.4 , which is the maximum value that the slope angle can attain when the rolling resistance torque equals the one produced by gravity acting on the particle.

In order to simulate the experimental set up, we first create a rough surface of glued spheres with diameter $500 \mu \mathrm{m}$. We randomly deposit up to three layers of these spheres on the surface. In all cases, they are sitting on a stable position under gravity with one contact in the case of the first layer, or up to three contacts for the two upper layers. Thereafter, all the spheres are assumed as fixed in their positions and motionless respect to the vibrating plate. They only interact with the upper moving spheres. This glued layer simplifies the global interaction calculation, as only sphere contacts are present in the simulation, i.e., no flat wall-sphere interactions are possible.

Once the rough surface is ready, 200 spheres with $1 \mathrm{~mm}$ diameter are randomly deposited on it to consider a large number of different configurations. To earn computing time, we prevent the particles to see each other, i.e., no billiard balls effects are present during all the simulation process. After the moving spheres attained equilibrium positions, the surface constituted by the glued spheres is put under vertical oscillation at a given amplitude and frequency by moving the center of these spheres. Due to the interaction forces, this process will consequently generate the oscillation of all the spheres. It is worthy to mention here that this oscillation will limit the choice of the time step for simulations. Indeed, besides the classical soft DEM rule of using the length of the time steps for the numerical integration as 
$1 / 50$ of the duration of the collisions, we have to take into account that the displacement provoking the oscillation of the glued spheres will generate an overlap with the upper spheres that has also to follow that soft DEM rule.

Since increasing the frequency step by step like in the experiments would consume a very long computer time, we run the program a few times for each frequency-amplitude pair and look for spheres in movement after a given time. Thus, we can easily get the $50 \%$ of moving spheres threshold with a good statistic. The set of input parameters used in the simulation are given in Table 1.

\subsection{Results and Discussion}

In Figure 10 we show the simulation results for the critical pair frequency-amplitude for the case of $500 \mu \mathrm{m}$ surface and glass beads with diameter $1 \mathrm{~mm}$. The corresponding experimental and theoretical results are shown for comparison. The exponential decay is recovered again in simulations and the agreement with experiments is reasonably good. The lines in the figure indicate fitting exponential curves.

We understand that the main reason for explaining the difference in behavior between simulations and experiments has to do with the roughness of the surface. In simulations, the number of layers and the mean distance between spheres in these upper layers do not have the topological complexity of the surface generated through real glued spheres. In this way, the ease of the moving beads to overcome the barrier to jump from a hole is greater for the case of simulations than for experiments. This is evident especially for lower amplitudes where the critical frequencies observed in simulations are respectively lower. We also try other ratios and confirm this behavior. This will be the subject of further research. 


\section{Conclusion}

In this work, we aim to determine the critical moment when particles deposited on a vibrating surface are put into movement. Although the experimental set up is rather simple, the explanation of the exponential decaying relation found for the critical frequencyamplitude pairs is not straightforward.

As discussed in previous works (Ziskind, Fichman \& Gutfinger (2000)), our experiments show that the frequencies are quite lower than those related to the elastic properties of the particles material $\left(6 \times 10^{6} \mathrm{rad} / \mathrm{s}\right.$ or higher). These frequencies follow an exponential decay with the amplitude of the sinusoidal external excitation.

Following the idea that particles behave like a forced damped oscillator, we develop a simple resonant spring model where the key point stands on the assumption that the stiffness of the vibrating spring encompasses all the moving particle interactions with the surface and it results to be quite lower than the one associated to glass stiffness alone. Besides, it is necessary to assume that the natural frequency of that spring depends on the particle deformation and, as a consequence, on the acceleration amplitude of the vibrations. This is in line with other authors' arguments, where the stiffness constants of oscillators are analyzed as a function of the external excitation for both linear and non-linear restoring forces (Ziskind, Fichman \& Gutfinger (2000); Kovacic (2011)). The idea of a harmonic oscillator resonating at the natural frequency corresponding to the particle material, alone, is not enough. In this way, the theoretical model proposed here describes the experimental behavior quite closely, explaining the exponential decay obtained for the critical frequencies, although it is not explicitly derived from the theoretical calculus. 
Contrary to the theoretical model, where the elastic constant can be thought of as the resultant of all the interactions of the particle with the surface, in the simulation model, the lack of a real topological description of the surface roughness is responsible that the critical frequencies result lower than expected, especially for lower amplitudes where space barriers are more difficult for the moving particles to overcome. In this sense, a deeper study of the critical frequency-amplitude pairs for incipient movement of particles on a highly controlled monolayer of glued spheres is in course.

\section{Appendix}

\section{Spring-dashpot model for particle contact}

The $i_{\text {th }}$ particle is characterized by its radius $\boldsymbol{r}_{i}$, the position of its center $\left(x_{i}, y_{i}\right)$ and the angular rotation $\theta_{i}$ around its center. Interparticle forces exist only when two spheres overlap or when the cohesive forces keep them in close contact, and the normal and tangential contact forces increase as the centers of the particles approach each other.

The normal force $F_{n}$ at the contact is modeled as viscoelastic. It consists of an elastic (a linear spring) and a viscous damping (a linear dashpot) contributions, described as follows:

Compression: $F_{n}=K_{n} \delta-b_{n} v_{n}$ for $\delta=\left(\sigma-\left|\boldsymbol{r}_{i}-\boldsymbol{r}_{j}\right|\right)>0$

Tension: $F_{n}=0$ for $\delta<0$

where $K_{n}$ is the spring constant for normal forces, $\delta$ is the relative normal displacement between the centers of the two particles in contact, $\sigma$ is the distance between their two 
centers, $\boldsymbol{r}_{i}$ and $\boldsymbol{r}_{j}$ are the two radii of the particles, $v_{n}$ is the relative normal velocity and $b_{n}$ is the dashpot constant for normal forces.

The force in the tangential direction is also modeled as a viscoelastic one where a linear spring and a linear dashpot are used as follows:

$$
F_{t}=K_{t} \delta_{t}-b_{t} v_{t}
$$

where $K_{t}$ is the spring constant for tangential forces, $\delta_{t}$ is the relative lateral displacement during all the duration of the contact, $v_{t}$ is the relative tangential velocity, and $b_{t}$ is the dashpot constant for tangential forces. $F_{t}$ is also limited to a maximum value which is chosen according to a Coulomb friction law where slipping can occur:

$$
F_{t}=\mu_{i} F_{n}
$$

where $\mu_{i}$ is the coefficient of friction. The tangential force acts in a direction opposite to that of the relative tangential velocity $v_{t}$.

The definition of the two spring constants and the two dashpot terms can be directly related to the classical mechanical parameters describing a material, i.e., Young's modulus, Poisson ratio and restitution coefficient (see, for example, Mishra \& Murty (2001)).

In the present model, we can introduce bonds between spheres that are in contact depending of the surrounding water content. The decrease of the cohesive force $\left(F_{c a p}\right)$ during the pulling apart process of two adjacent spheres is modeled using the equation proposed by Charlaix \& Crassous (2005):

$$
F_{c a p}=2 \pi \gamma_{s l} R \cos (\theta)
$$


where $\cos (\theta)$, defined from the wetting angle, is around 0.84 for water on glass, $\gamma_{s l}$ is the liquid surface tension and $R$ is the effective radius of the two interacting particles.

Finally, the van der Waals force between particles, $F_{V W}$, is modeled through an always attractive force as (Israelachvili (1991)):

$$
F_{V W}=\frac{A_{H} R}{6 D^{2}}
$$

where $A_{H}$ is the Hamaker coefficient, $R$ is, as in (A4), the effective radius and $\mathrm{D}$ is the distance between the surfaces.

\section{Acknowledgements}

Authors want to acknowledge financial support from Universidad Nacional de San Luis through PROICO 310114, from CONICET through PIP 353, from MYNCyT-ECOS Sud, Argentine-France, project A15E03 and LIA CONICET-CNRS Physique et Mécanique de Fluides.

\section{References}

Ai, J., Chen, J.-F., Rotter, J. M., \& Ooi, J. Y. (2011). Assessement of rolling resistance models in discrete element simulations. Powder Technology, 206, 269-282.

Bagnold, R.A. (1941). The Physics of Blown Sand and Desert Dunes. Chapman \& Hall (p 265).

Charlaix, E. \& Crassous, J. (2005). Adhesion forces between wetted solid surfaces. The Journal of Chemical Physics, 122, 184701. 
Chepil, W.S. (1945). Dynamics of wind erosion: I. Nature of movement of soil by wind. Soil Science, 60, 305-320.

Dabros, T.,Warszynsky, P., \& van de Ven, T. G. N. (1994). Motion of latex spheres tethered to a surface. Journal of Colloid Interface Science, 162, 254-256.

D'Anna, G., \& Gremaud, G. (2001). Vibration-induced jamming transition in granular media. Physical Review E, 64, 011306.

Dybwad, G. L. (1985). A sensitive new method for the determination of adhesive bonding between a particle and a substrate. Journal of Applied Physics, 58, 2789-2790.

Eshuis, P., van der Weele K., van der Meer, D., Bos R., \& Lohse, D. (2007). Phase diagram of vertically shaken granular matter. Physics of Fluids, 19, 123301.

Herminghaus, S. (2005). Dynamics of wet granular matter. Advances in Physics, 54, 221261.

Hein, K., Hucke, T., Stintz, M., \& Ripperger, S. (2002). Analysis of adhesion forces between particles and wall based on the vibration method. Particle \& Particle Systems Characterization, 19, 269-276.

Henry, C., \& Minier, J.-P. (2014). Progress in particle resuspension from rough surfaces by turbulent flows. Progress in Energy and Combustion Science 45, 1-53.

Herrmann, H. J., \& Luding, S. (1998). Modeling granular media with the computer. Continuum Mechanics and Thermodynamics, 10, 189-231.

Israelachvili, J.N., 1991. Intermolecular and Surface Forces. Academic Press, London.

Jones, R., Pollock, H.M., Cleaver, J.A.S., \& Hodges, C.S. (2002). Adhesion Forces between Glass and Silicon Surfaces in Air Studied by AFM: Effects of Relative Humidity, Particle Size, Roughness, and Surface Treatment. Langmuir 18, 8045-8055.

Kohonena, M. (2004). On capillary bridges in wet granular materials, Physica A, 339, 7-15. 
Kovacic, I. (2011). Forced vibrations of oscillators with a purely nonlinear power-form restoring force. Journal of Sound and Vibration, 330, 4313-4327.

Luding, S. (1998). Collisions \& contacts between two particles. In: H. J. Herrmann, J.-P. Hovi, \& S. Luding (Eds.), Physics of dry granular media, (p. 285). NATO ASI Series E350, Kluwer Academic Publishers.

Mawatari, Y., Tatemoto, T. K. Y., Uchida, S., \& Noda, K. (2002). Effect of particle diameter on fluidization under vibration. Powder Technology, 123, 69-74.

Melo, F.,Umbanhowar, P. B., \& Swinney, H. L. (1994).Transition to parametric wave patterns in a vertically oscillated granular layer. Physical Review Letters, 72, 172-175.

Melo, F., Umbanhowar, P. B., \& Swinney, H. L. (1995). Hexagons, kinks, and disorder in oscillated granular layers. Physical Review Letters, 75, 3838-3841.

Metcalf, T. H., Knight, J. B., \& Jaeger, H. M. (1997). Standing wave patterns in shallow beds of vibrated granular material. Physica A, 236, 202-210.

Mishra, B.K., \& Murty, C.V.R. (2001). On the determination of contact parameters for realistic DEM simulations of ball mills. Powder Technology, 115, 290-297.

Mullins, M. E., Michaels, L. P., Menon, V., Locke, B., \& Ranade, M. B. (1992). Effect of geometry on particle adhesion. Aerosol Science and Technology, 17, 105-118.

Oger, L., Ippolito, I., \& Vidales, A. M. (2007). How disorder can diminish avalanche risks: effect of size distribution. Granular Matter, 9, 267-278.

Oger, L., \& Valance, A. (2017). Model of the saltation transport by Discrete Element Method coupled with wind interaction, European Physical Journal Web of Conferences, $140,12004$.

Pain, H.J. (1976). The physics of vibrations and waves (p. 300). J. Wiley \& Sons (2 ${ }^{\text {nd }}$. Edition). 
Rabinovich, Y. I., Adler, J., Esayanur, M. S., Ata, A., Singh, R., \& Moudgi, B. (2002). Capillary forces between surfaces with nanoscale roughness. Advances in Colloid and Interface Science, 96, 213-230.

Reeks, M. W., \&Hall, D. (2001) Kinetic models for particle resuspension in turbulent flows: theory and measurement. Journal of Aerosol Science, 32, 1-31.

Renard, S., Schwager, T., Pöschel, T. \& Salueña, C. (2001). Vertically shaken column of spheres. Onset of fluidization. Europhean Physical Journal E, 4, 233-239.

Ripperger, S., \& Hein, K. (2005). Measurement of adhesion forces in air with the vibration method, China Particuology, 3, 3-9.

Savage, S. B. (1993). Disorder, diffusion, and structure formation in granular flows. In: D. Bideau (Ed.), Disorder and granular media (p. 264). North Holland, Amsterdam.

Soepyan, F., Cremaschi, S., McLaury, B., Sarica, C., Subramani, H., Kouba, G., \& Gao, H. (2016). Threshold velocity to initiate particle motion in horizontal and near-horizontal conduits. Powder Technology, 292, 272-289.

Theerachaisupakij, W., Matsusaka, S., Kataoka, M., \& Masuda, H. (2002). Effects of wall vibration on particle deposition and reentrainment in aerosol flow. Advanced Powder Technology, 13, 287-300.

Tippayawong, N., \& Preechawuttipong, I. (2011). Analysis of microparticle resuspension in turbulent flows with horizontally vibrating surface. Australian Journal of Basic and Applied Sciences, 5, 356-363.

Valenzuela Aracena, K.A., Benito, J. G., Oger, L., Uñac, R. O., Ippolito, I., \& Vidales, A. M. (2017). Experiments and numerical modeling for the movement and resuspension of grains. European Physical Journal Web of Conferences, 140, 03014. 
Wang L. (1999). The role of damping in phase imaging in tapping mode atomic force microscopy, Surface Science, 428, 178-185.

Zingg, A.W. (1953). Wind tunnel studies of the movement of sedimentary material. Fifth Hydraulics Conference Proceedings, J.S. McNown, \& M.C. Boyer (Eds.), 111-136.

Ziskind, G., Fichman, M., \& Gutfinger, C. (2000) Particle behavior on surfaces subjected to external excitations. Journal of Aerosol Science, 31, 703-719.

Zhu, H.P., Zhou, Z.Y., Yang, R.Y., \& Yu, A.B. (2007). Discrete particle simulation of particulate systems: Theoretical developments. Chemical Engineering Science, 62, 33783396.

Table

Table 1: Input parameters for the $500 \mu \mathrm{m}$ surface and $1 \mathrm{~mm}$ moving glass beads at $70 \%$ of relative humidity

\begin{tabular}{|l|l|}
\hline Parameter & Value \\
\hline Density for glass & $2500 \mathrm{~kg} / \mathrm{m}^{3}$ \\
\hline Friction coefficient, $\mu_{i}$ & 0.5 \\
\hline Restitution coefficient & 0.8 \\
\hline Stiffness, $K_{n}$ & $10^{6} \mathrm{~N} / \mathrm{m}$ \\
\hline Stiffness, $K_{t}$ & $1.6 \times 10^{6} \mathrm{~N} / \mathrm{m}$ \\
\hline Dashpot constant, $b_{n}$ & $0.11 \mathrm{~kg} / \mathrm{s}$ \\
\hline Dashpot constant, $b_{t}$ & $0.4 b_{n}$ \\
\hline Rolling resistance & 0.3 \\
\hline Surface tension, $\gamma_{s l}$ & $72 \mathrm{mN} / \mathrm{m}$ \\
\hline Hamaker coefficient, $A_{H}$ & $6.5 \times 10^{-20} \mathrm{~J}$ \\
\hline
\end{tabular}

\section{Figure captions}




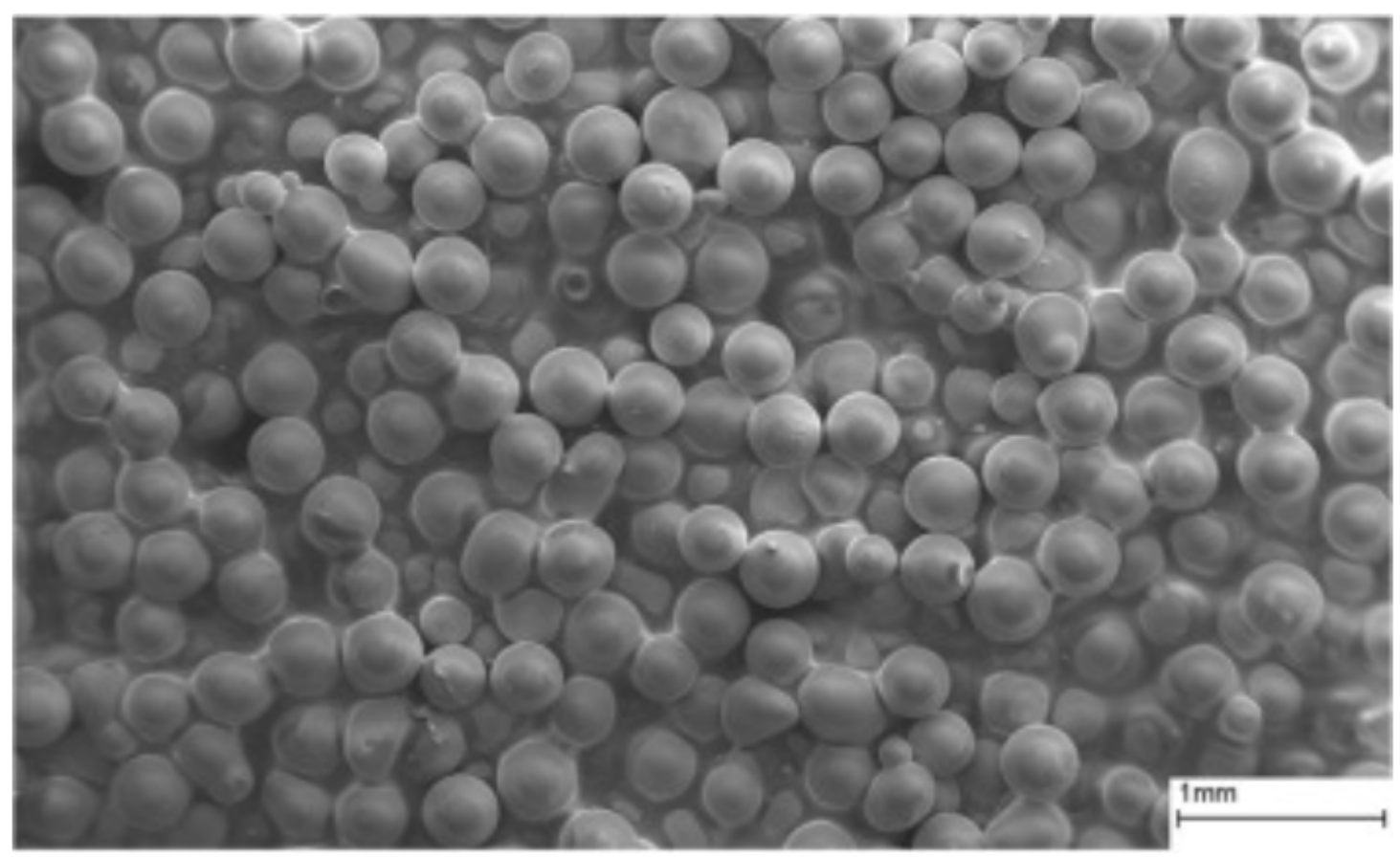

Figure 1: SEM image from the surface of $250 \mu \mathrm{m}$ glass beads.

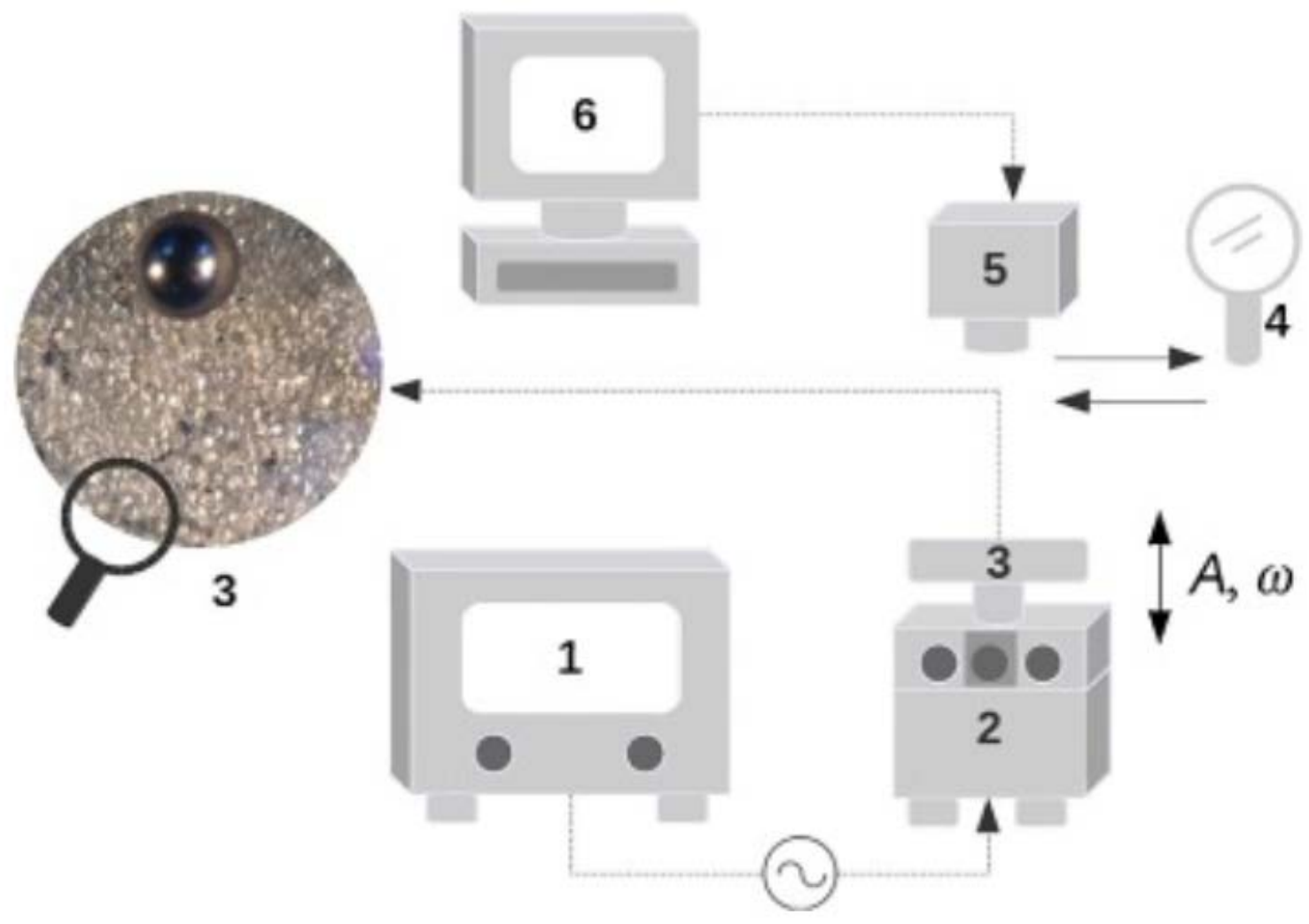


Figure 2: Sketch of the experimental set up used to measure the critical amplitudefrequency pairs: Sine wave generator (1), Mechanical wave driver (2), Rough surface(3), Loupe (4), Digital camera (5), Computer (6).
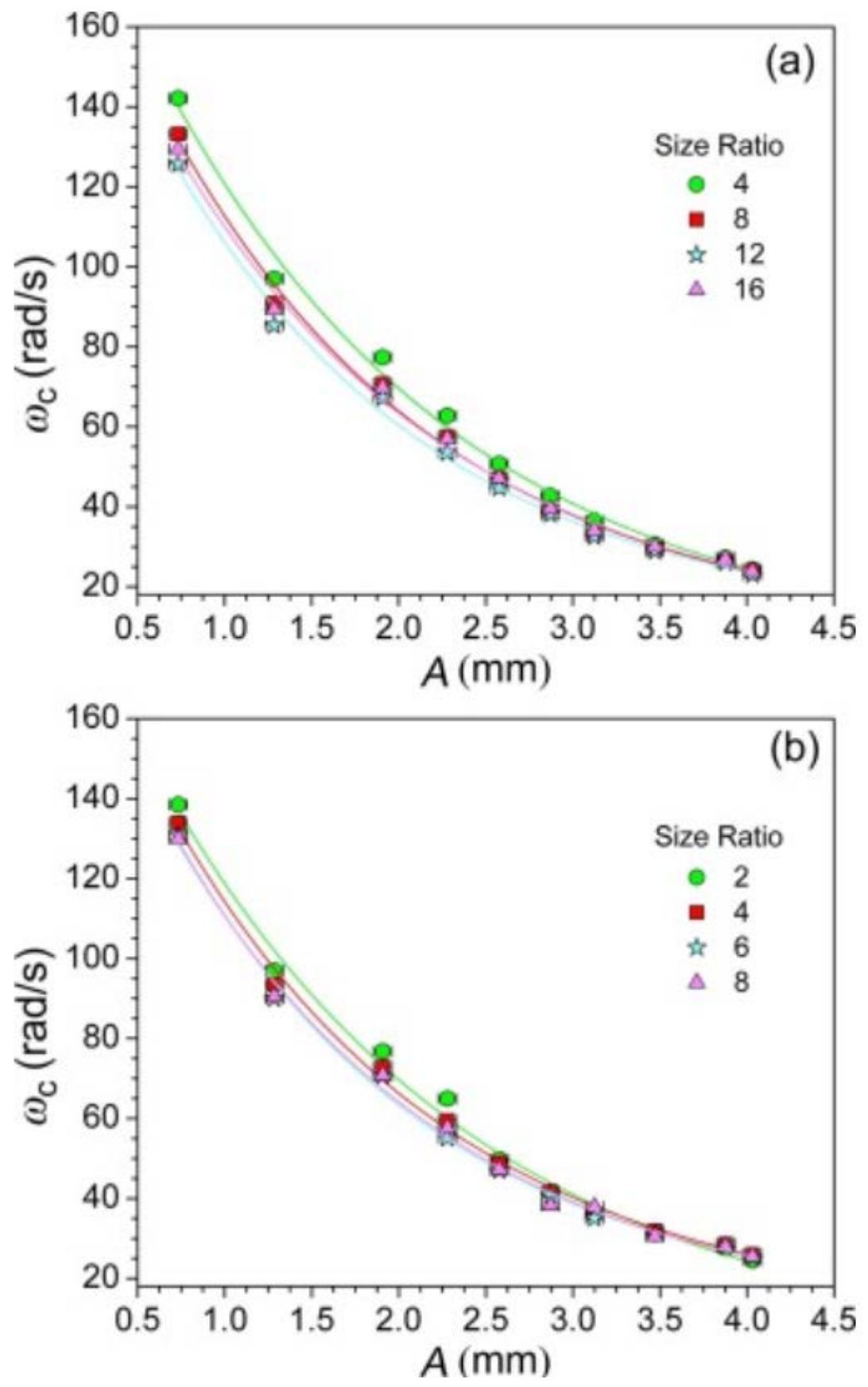

Figure 3: Critical frequency as a function of the vibration amplitude for glass beads on (a) $250 \mu \mathrm{m}$ and (b) $500 \mu \mathrm{m}$ glass surface. The size ratios indicated correspond to the ratio 
between the diameter of the free spheres and the diameter of the beads belonging to the surface. The error bars are indicated. The curves correspond to exponential fits.
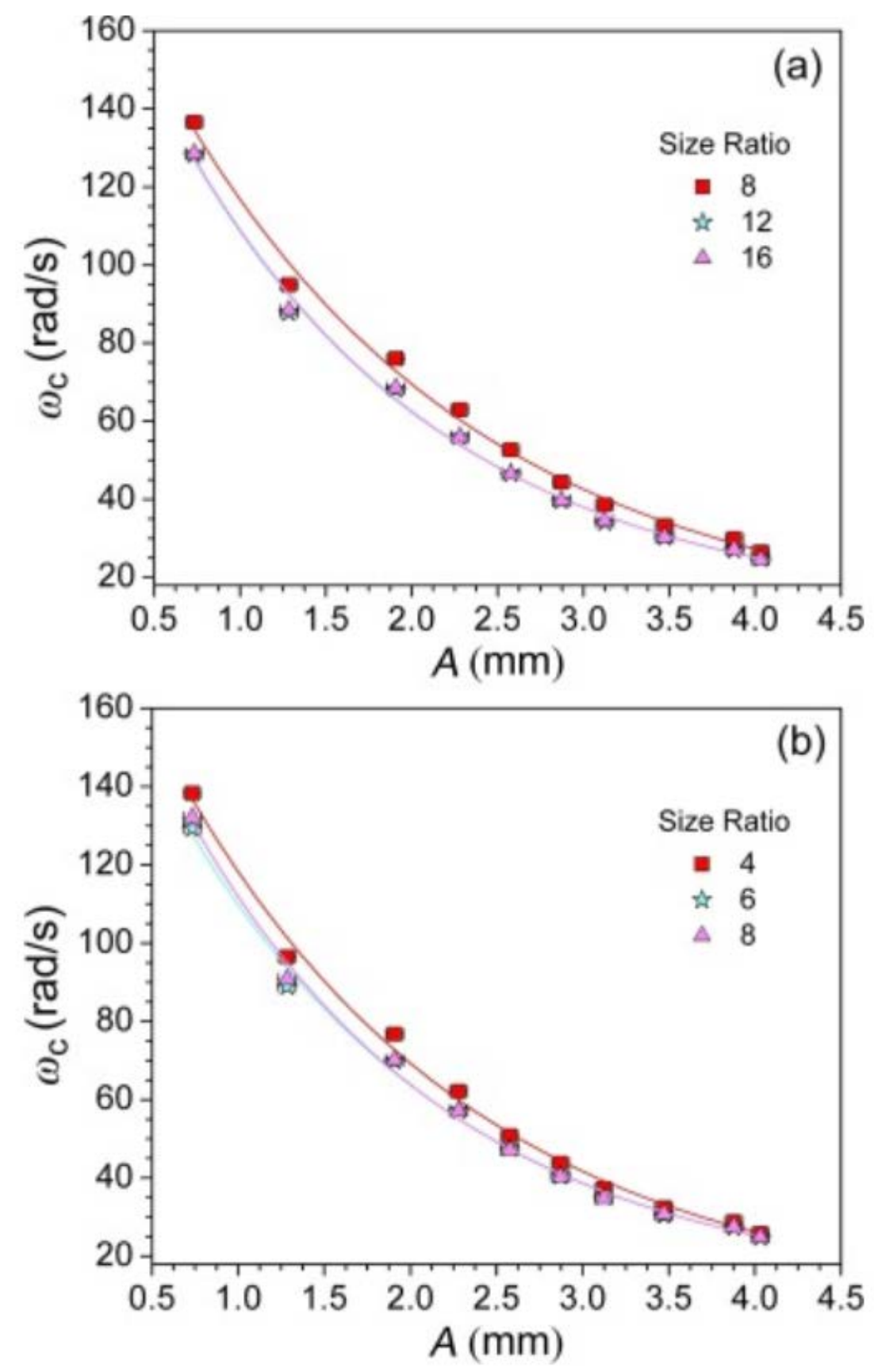

Figure 4: Critical frequency as a function of the vibration amplitude for stainless steel beads on (a) $250 \mu \mathrm{m}$ and (b) $500 \mu \mathrm{m}$ glass surface. The size ratios indicated correspond to 
the ratio between the diameter of the free spheres and the diameter of the beads belonging to the surface. In both cases, the error bars are indicated and, as before, the curves are exponential fits.

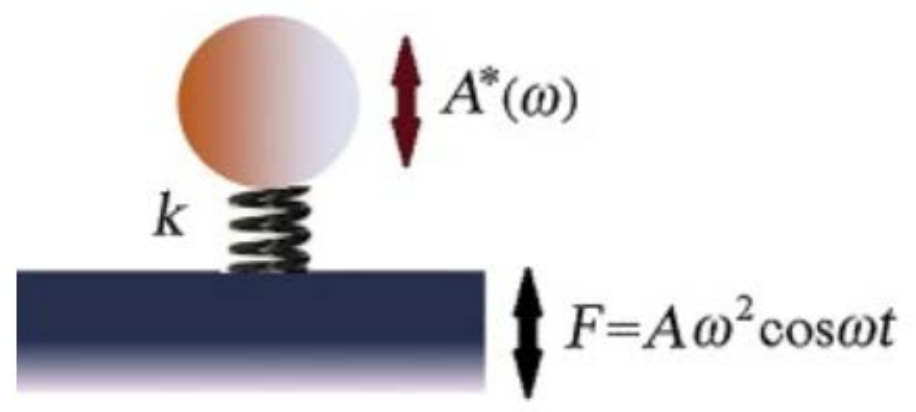

Figure 5: Sketch of the forced damped harmonic oscillator representing our model system.

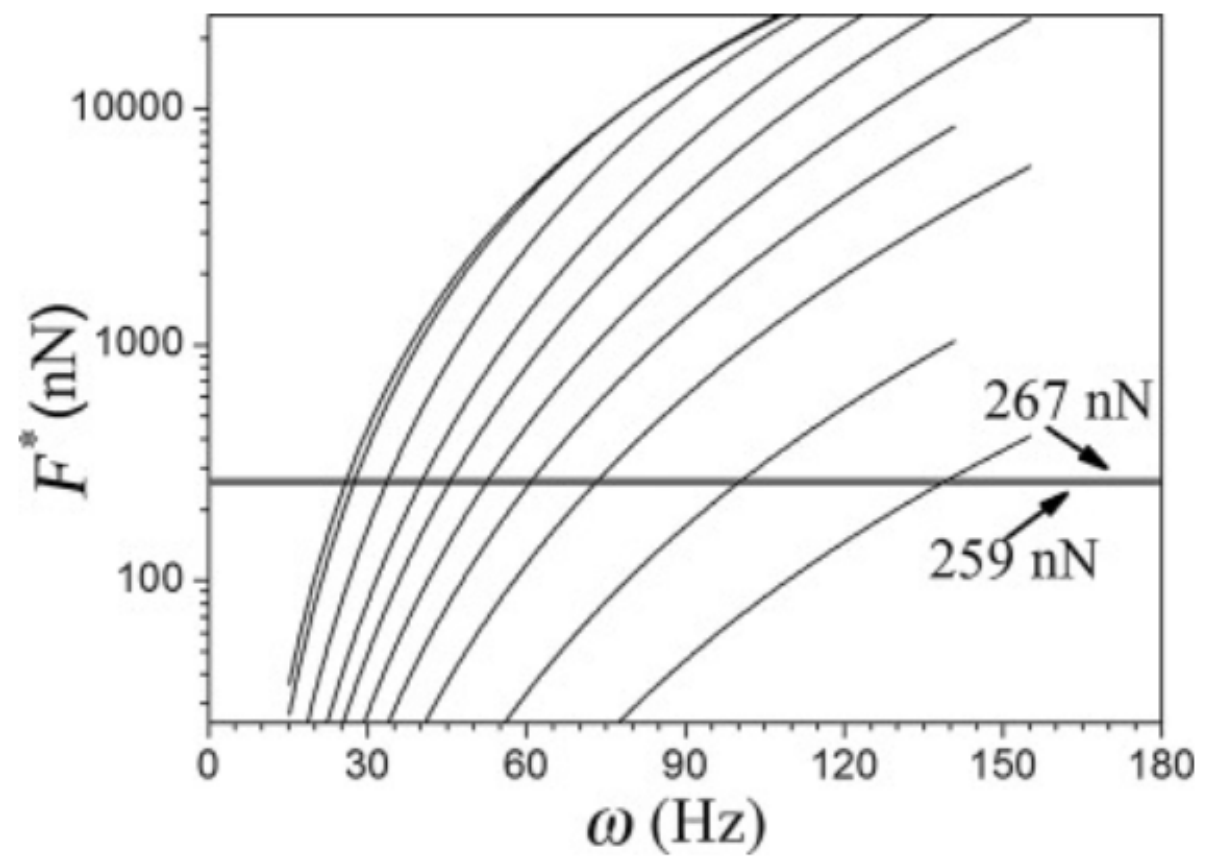

Figure 6: Calculated forces undergone by the particle deposited on the oscillating surface and for different amplitudes, which (from left to right) are: 0.73, 1.28, 1.91, 2.28, 2.58, 2.87, 3.12, 3.47, 3.88, 4.03 millimeters. Note the semi-log scale. The horizontal lines correspond to the detaching forces for each surface: $250 \mu \mathrm{m}$ (upper) and $500 \mu \mathrm{m}$ (lower). 


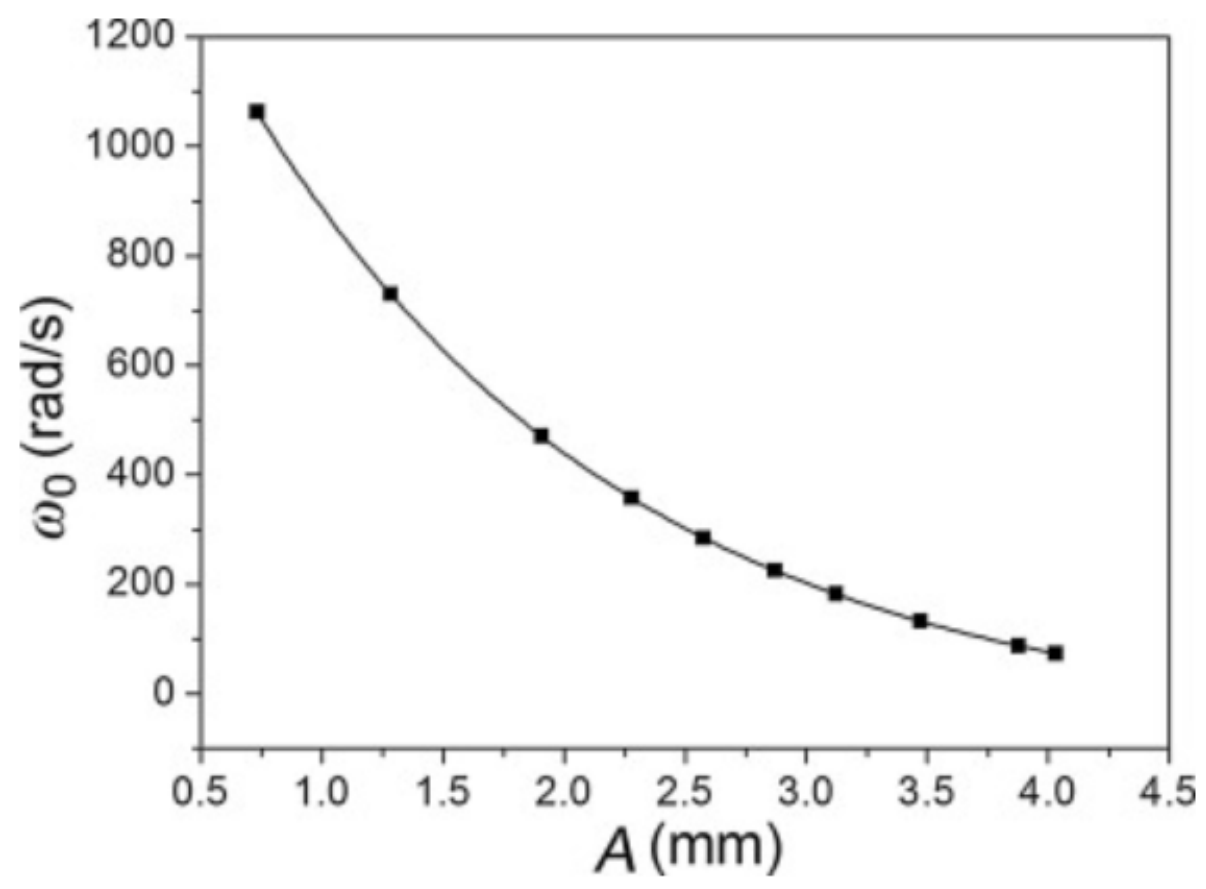

Figure 7: Exponential decay assumption for the dependence of the natural frequency, $\omega_{0}$, with the amplitude, A. The points correspond to the values used in the theoretical calculations for both types of surfaces. 

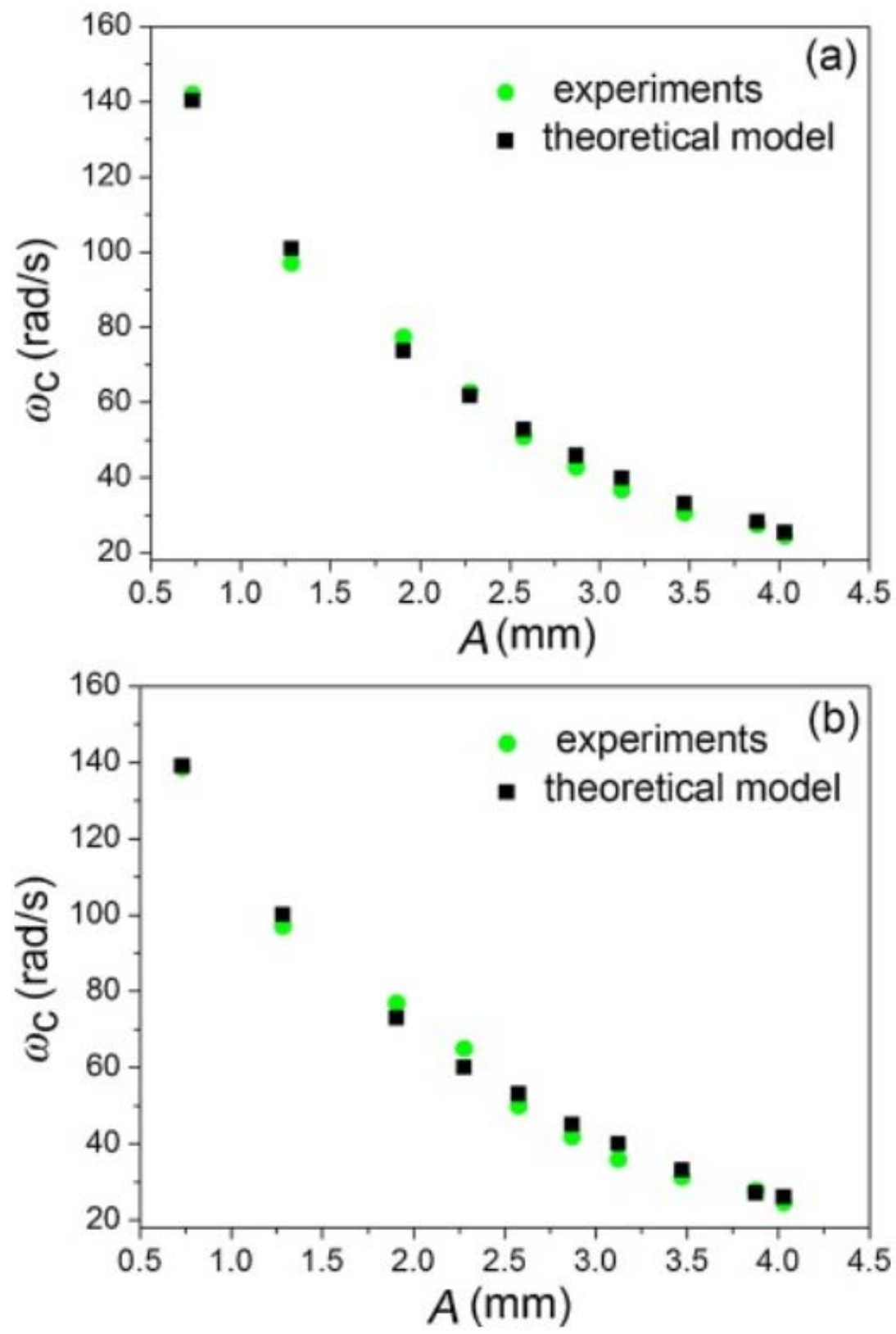

Figure 8:Comparison between the experimental and theoretical values for the critical frequency-amplitude pairs for incipient movement of a $1 \mathrm{~mm}$ glass sphere over: (a) $250 \mu \mathrm{m}$ and (b) $500 \mu \mathrm{m}$. 

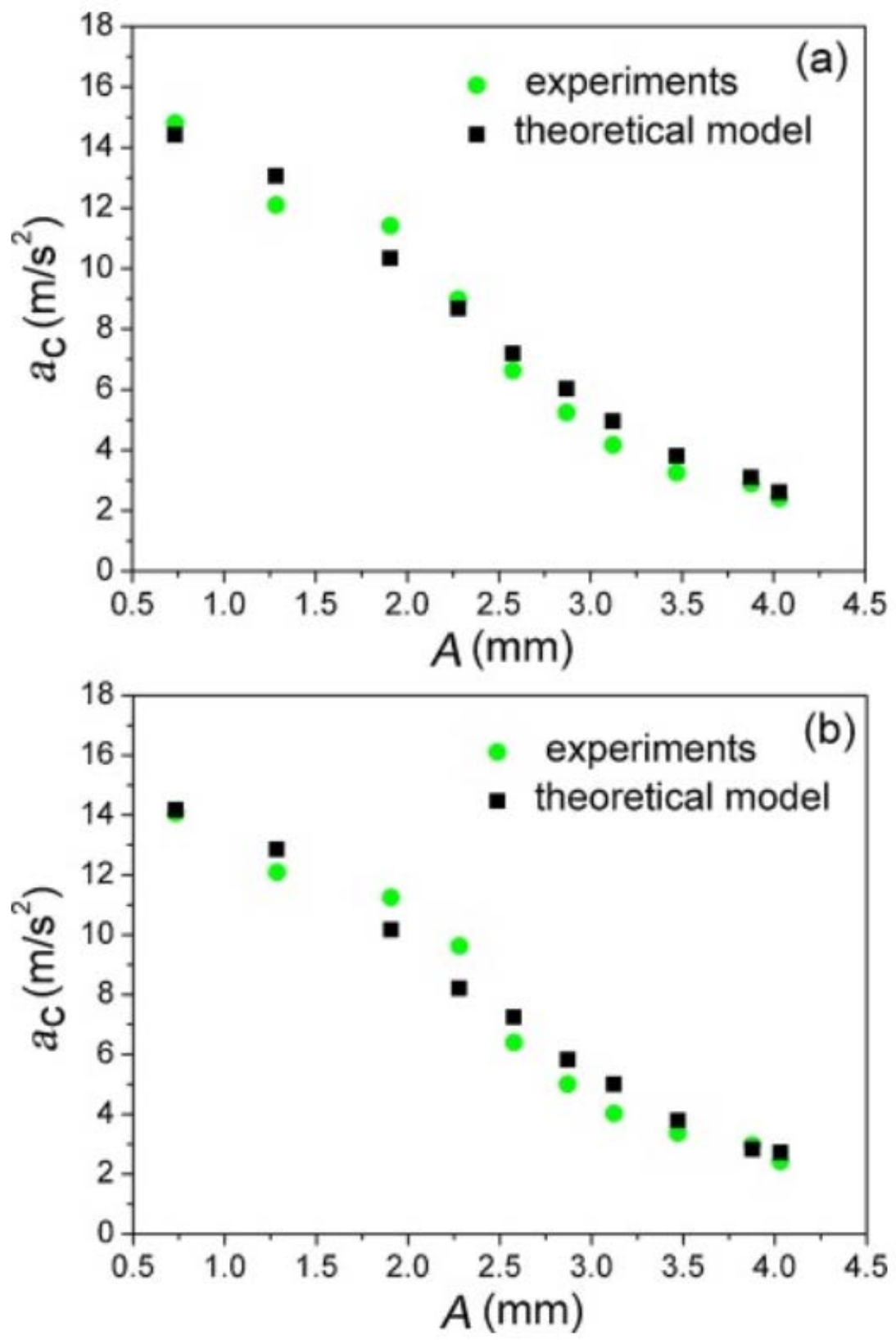

Figure 9: Comparison between the experimental and theoretical values for the maximum critical acceleration at which the vibrating plate is subjected when particle movement is starting for a $1 \mathrm{~mm}$ glass sphere over: (a) $250 \mu \mathrm{m}$ and (b) $500 \mu \mathrm{m}$. 


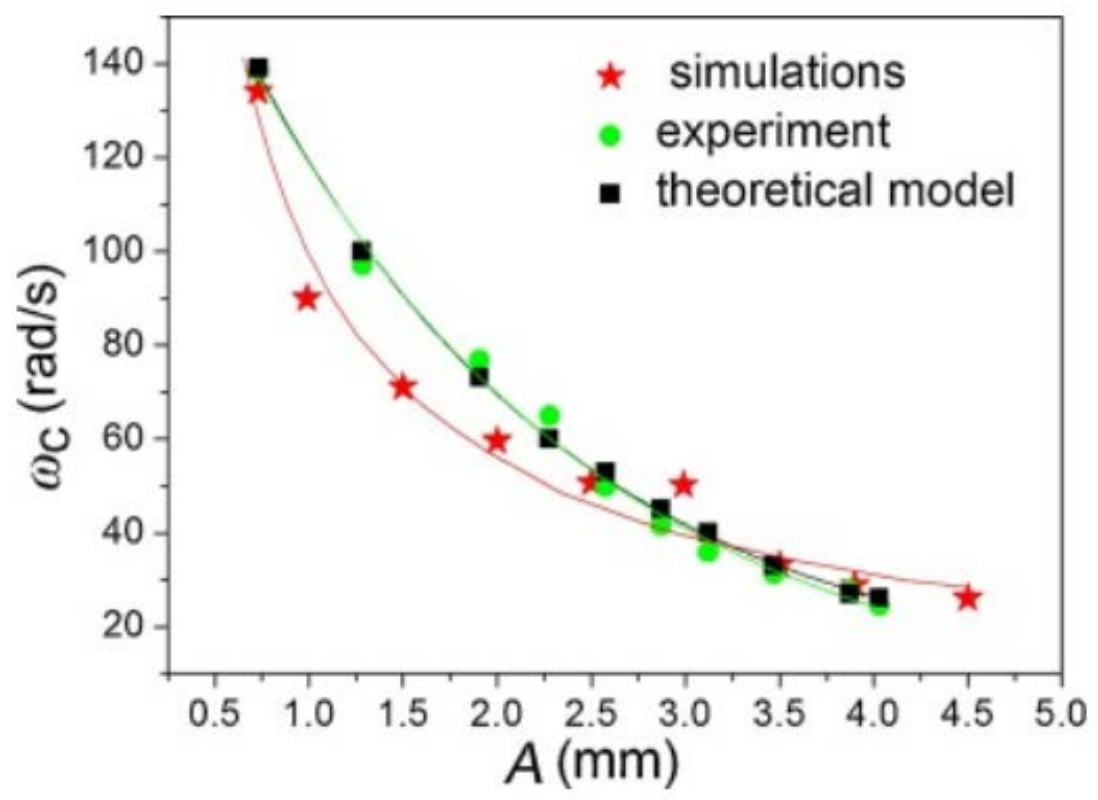

Figure 10: Comparison with simulation results for critical frequency-amplitude pairs for the case of a surface of $500 \mu \mathrm{m}$ glass beads and $1 \mathrm{~mm}$ moving particles. 